


\section{EL PRINCIPIO DEL DETERMINISMO EN EL MATERIALISMO CULTURAL 1}

(Recepción: Junio 26 de 2013- Aceptación: Agosto 27 de 2013)

Ramiro Ceballos Melguizo*

Óscar Javier Cabeza Herrera **

El autoengaño y la subjetividad no son medidas de lo humano.

(Marvin Harris)

\section{Resumen}

El principio del determinismo tecno-ecológico y tecno-económico constituye el núcleo explicativo del materialismo cultural de Marvin Harris. El presente artículo describe y analiza este concepto, se reflexiona sobre su significado y sobre las objeciones que es posible hacerle. Las principales objeciones son dos: la posible negación de la libertad que implicaría, y la dificultad que conlleva la impredecibilidad de la acción humana.

\section{Palabras Clave}

Antropología, Cultura, Determinismo, Libertad, Materialismo.

\section{THE DETERMINISM PRINGIPLE IN CULTURAL MATERIALISM}

\section{Alustract}

The principle of techno-ecological and techno-economic determinism constitutes the explanatory core of Marvin Harris's cultural materialism. This article describes and analyzes this concept, reflects on its significance and possible objections to it. There are two main objections: the potential denial of freedom that it would entail, and the difficulties inherent to the unpredictability of human action.

\section{Keywords}

Anthropology, Culture, Determinism, Freedom, Materialism.

1 Artículo de investigación filosófica, desarrollado en la Facultad de Artes y Humanidades de la Universidad de Pamplona en el año 2013. Responde a la línea de investigación en Filosofía y Pedagogía: -Proyecto Epistemología y Antropología- del grupo de investigación CONQUIRO, inscrito en Colciencias con el código COL014098120130419859 del Departamento de Filosofía de la Universidad de Pamplona.

* Ramiro Ceballos Melguizo es docente-investigador del grupo Conquiro del Departamento de Filosofía, Universidad de Pamplona. Colombiano. Filósofo y Magíster en Filosofía de la Universidad de Antioquia. Profesor Tiempo Completo, Facultad de Artes y Humanidades, Universidad de Pamplona, Colombia. E-mail: ramirocem@yahoo.es

** Óscar Javier Cabeza Herrera es docente-investigador del grupo Conquiro del Departamento de Filosofía, Universidad de Pamplona. Colombiano. Licenciado en Filosofía e Historia, Universidad Santo Tomás; Especialista en Cultura Política y Educación, Universidad de Pamplona; Magíster en Bioética, Universidad El Bosque. Profesor Tiempo Completo, Facultad de Ciencias de la Educación, Universidad de Pamplona, Colombia. E-mail: oscar.cabeza@hotmail.com 


\section{Introducción}

El presente escrito constituye el resultado académico de la indagación permanente del grupo de investigación Conquiro, el cual toma como referente la tradición del pensamiento y el quehacer filosófico, como punto de convergencia para la labor docente, siempre con el objeto de construir conocimiento crítico que fortalezca la investigación formativa propia a la labor pedagógica y didáctica del Departamento de Filosofía de la Universidad de Pamplona. La línea de investigación a la que pertenece el presente proyecto -Epistemología y Antropología-, quiso abordar críticamente al materialismo cultural, cuyo fundamento último y estrategia de investigación consiste en reclamar para la antropología el derecho a que sus resultados sean asumidos conforme a los criterios de la ciencia, se reafirma así la prioridad metodológica de la búsqueda de leyes en el estudio del hombre.

No ha sido Marvin Harris el primero ni el único que se ha propuesto hacer de la antropología una disciplina capaz de formular explicaciones de carácter general y de validez universal; la misma pretensión inspiraba ya a Malinowski y a Radcliffe Brown, para citar solamente a dos ilustres representantes del funcionalismo y el estructuralismo, cuyos programas de investigación contenían perspectivas epistemológicas opuestas al particularismo histórico (Franz Boas) y, en general, a una tendencia con fuertes propensiones relativistas que dominó los estudios antropológicos a comienzos de siglo $X X$.

Sin embargo, ha sido Harris quien con mayor claridad ha sostenido el ideal nomotético de una teoría de la cultura, orientando la investigación etnológica en la perspectiva de la ciencia empírica y desmarcándose, en primer lugar, de las tendencias ideográficas y hermenéuticas de la interpretación de los hechos humanos.

La idea de hacer extensivos al dominio de los hechos culturales los modos de conocimiento de las ciencias naturales se consideró siempre una actitud gnoseológicamente reprensible. Bajo el epíteto de "cientifismo" se le ha descalificado a menudo, argumentando que el orden de los sucesos característicos de la acción humana está expuesto a la interferencia azarosa de la libre elección, por lo que habrá de resultar vano e ilusorio cualquier intento de generalización que pretenda adecuarse a los modelos predictivo y retrodictivo, que son el sustento metodológico privativo de las ciencias físico-naturales.

Puede decirse que la totalidad de las ciencias humanas, o ciencias del espíritu, según la terminología de Dilthey y otros, han nacido en lucha por superar la negatividad que comparte el anterior argumento. Al mismo tiempo, esta negatividad se ha combatido muchas veces a costa de la propia unidad de la razón y/o en detrimento de la legalidad de los objetos que se pretende abordar. En lo que atañe a la antropología, el siglo de las luces ha representado el periodo de conquista y gestación del principio teórico que permite superar la dificultad epistémica a la que se enfrenta una ciencia de la cultura. Los ilustrados reafirmaron la racionalidad de los hechos históricos en general y, en consecuencia, aunque no lograron superar el dilema entre cognoscibilidad nomotética y libre albedrío en el dominio de los hechos humanos, se los puede considerar precursores directos de la antropología en cuanto ciencia del hombre (Harris, 1981, pp.7-45).

El materialismo cultural, programa de investigación cuyo modo de conocimiento pretende fundarse en la ciencia empírica, es tributario de esta pretensión 
"cientifista", de la cual, afirma Harris, recupera la forma, pero no la sustancia. Esto es posible gracias a la adopción de un principio macro-teórico de la evolución cultural ${ }^{2}$. Se trata del principio del determinismo tecno-ecológico y tecno-económico, el cual es concebido como un análogo del principio darwiniano de la selección natural, por el que se orientará la teorización en la perspectiva de la mayor coherencia y la máxima simplicidad en las explicaciones, sin que ello conduzca, por un reduccionismo inevitable, el acotamiento ontológico de las realidades constitutivas de la cultura, conforme a los modelos apriorísticos justamente criticados desde la estrategia materialista cultural.

\section{El Método}

La invocación de los modos de conocimiento de la ciencia empírica constituye el fundamento metodológico del materialismo cultural. Por otro lado, como suscriptor del principio materialista, esta estrategia antropológica sólo necesita afirmar que las entidades materiales tienen una existencia propia, separada de las ideas. Su problema central no consiste entonces en demostrar la tesis de la constitución material última de las cosas, sino desarrollar mecanismos que permitan obtener conocimiento válido tanto del dominio de las entidades materiales como del dominio de las ideas, las cuales constituyen elementos correlativos del objeto real, esto es, de la cultura.

La primera decisión metódica del materialismo cultural consiste en adoptar un par de distinciones: I) entre sucesos mentales y conductuales y 2) entre acontecimientos de tipo "Etic" y de tipo "Emic". El dominio mental y conductual

2 "Tecnologías similares aplicadas a medios similares tienden a producir una organización del trabajo similar, tanto en la producción como en la distribución y ésta, a su vez, agrupamientos sociales similares de valores y de creencias". (Harris, 1981, p.3). se especifican entonces, en relación con las operaciones diferentes que es preciso realizar para obtener afirmaciones válidas en torno a ellos. Ambos dominios pueden enfocarse en dos perspectivas distintas: Desde la de los propios participantes y desde la óptica del observador de un suceso determinado. En ambos casos es posible una descripción objetiva del fenómeno. El rasgo que distingue las operaciones de tipo "Etic" y de tipo "Emic" es la elevación del observador o del actor, respectivamente, a la categoría de juez de la adecuación de las descripciones y análisis.

No obstante, el materialismo cultural privilegia la perspectiva "Etic", otorgando completa autonomía a los recursos conceptuales usados por el observador y haciendo depender su validez no de la recurrencia de fenómenos "Emic" sino de su propia condición de elementos operativos exitosos en un corpus de teorías científicas.

\section{Principios teóricos}

La primera cuestión de principios atañe al modo de conceptualizar la naturaleza de las sociedades humanas. Contra los hábitos funcionalistas y estructuralistas, que dan por válido a priori el carácter sistemático de las organizaciones sociales, para el materialismo cultural esto es sólo un presupuesto estratégico que es preciso justificar mediante contrastaciones. En este sentido, el materialismo cultural se interesa por comprender causalmente las relaciones entre las partes de aquellos sistemas y por su evolución, pero sin postular modelos apriorísticos o, en todo caso, se especifica la índole epistémica de tales modelos como quiera que son, funcionalmente, principios taxonómicos para el control teórico de las explicaciones y muy impropiamente se puede intentar legitimarlos como invariantes cuyos correlatos ónticos serían evidenciables por vía intuitiva. Por el contrario, las 
explicaciones basadas en modelos taxonómicos deberán evaluarse en calidad de hipótesis "audaces" 3 y sujetas a contraste con los hechos.

El patrón universal del materialismo cultural, según el cual se conceptualiza la estructura de los sistemas socioculturales, se basa en constantes biológicas y psíquicas de la naturaleza humana, en las distinciones entre pensamiento $y$ conducta, y entre las perspectivas "Etic" y "Emic". Su modelo categorial es una versión revisada del modelo marxista: como categorías conductuales "Etic" los modos de producción y reproducción conformarán la infraestructura cultural; la estructura la constituyen el conjunto de prácticas ordenadas bajo los denominativos de "economía doméstica" y "economía política"; y la superestructura conductual la conformarían el conjunto de prácticas cuyos resultados constituyen los credos, ideologías y manifestaciones espirituales en general.

Paralelamente a estos sucesos conductuales se desarrollan los sucesos mentales, en calidad de componentes "Emic" de la estructura sociocultural. EI lenguaje no se conceptúa como categoría "Etic" o "Emic". Es un elemento cuyo tratamiento está implícito en las operaciones "Etic" y subtiende el proceso de interpretación de todos los acontecimientos comunicativos.

El otro principio clave del materialismo cultural consiste en la adopción de un análogo del principio marxista de la determinación de la conciencia social por el ser social: "Los modos de producción y reproducción conductuales "Etic" determinan probabilísticamente las economías doméstica y política conductuales "Etic", que a su vez determinan las superestructuras conduc-

3 La "Audacia" puede permutarse por las nociones de simplicidad y alcance o contenido de información, queda así definida en términos popperianos. tual y mental "Emic"." (Harris, 1982, p.72). El principio fundamental del materialismo cultural es, obviamente, el determinismo tecno-ecológico y tecno-económico, mencionado más arriba. A este principio, lo mismo que a sus implicaciones, irán dirigidas las reflexiones siguientes.

\section{Primacía de la infraestructura}

Estratégicamente, el primado de la Infraestructura significa otorgar la mayor prioridad en la formulación de teorías explicativas a los factores ecológicos, químicos y físicos a los que está sujeta la acción humana; al propio tiempo implica una voluntad de postergación de las explicaciones basadas en procesos superestructurales y mentales.

La justificación de este primado se apoya en la caracterización de la infraestructura como "zona interfacial", es decir, como límite y frontera entre cultura y naturaleza, donde se produce la interacción entre los acontecimientos reales y mensurables, a título de hechos naturales, y las prácticas socioculturales.

Esta decisión estratégica promete la construcción de teorías que incorporen regularidades sujetas a leyes de la naturaleza; de igual forma, indica el comienzo de cadenas causales que afectan la evolución sociocultural. En compensación por este "beneficio", la elección materialista se ve compelida a reducir las explicaciones hasta niveles inferiores de hechos mensurables, es decir, hasta el complejo de actividades orgánicas consumidoras de energía. La masa enorme de diferenciaciones de conducta que definen la cultura y la civilización habrá de ser proyectada en la dirección del principio del equilibro termodinámico, en cuanto instancia última de reducción, que, al mismo tiempo, poseería la ventaja de ser matemáticamente controlable y cumpliría así con uno de los criterios más 
apreciados por la perspectiva naturalista de explicación científica.

Ahora bien, para dar cuenta de modo satisfactorio de la emergencia de la cultura y de su naturaleza singular hay que determinar los procesos de selección responsables de la convergencia y divergencia de las trayectorias evolutivas de los sistemas socioculturales. Como estos procesos son vehiculizados por agentes individuales y mediados por la subjetividad, habrá que incardinar en el contexto del sistema cultural el conjunto de relaciones estructuradas que responden por la organicidad y por la dinámica de las sociedades. Como no se trata de proyectar ningún esquematismo a priori es necesario buscar una especie de media racional de los intercambios conductuales sobre la base de una interdependencia de motivos pragmáticos que fluctuaría regularmente entre los extremos relativos de lo individual y lo colectivo. "Hay que establecer el vínculo entre elecciones conductuales realizadas por individuos y las respuestas colectivas de los sistemas socioculturales" (Harris, 1982, p.76).

La posibilidad de salvar el abismo entre naturaleza y cultura, por un lado, y entre individuo y colectividad, por el otro, está condicionado entonces por la postulación de constantes biopsicológicas, las cuales habrán de permitir explicar cómo media la infraestructura entre cultura y naturaleza. Este problema, sin lugar a dudas, el más vasto y agudo de los problemas a que se hallan abocadas las ciencias del espíritu, reviste un especial carácter apremiante en la antropología. Como un primer paso para encararlo, Harris propone la construcción puntual y nomotética de una naturaleza humana a partir de universales psicobióticos, obviamente, con significado funcional antes que tético. Serían algunos de ellos: El principio de la optimización de nutrientes; el de la optimización de la acción motriz básica; el uso frecuente del goce sexual y el afán de reconocimiento.

\section{El carácter paradigmático del materialismo cultural}

Por oposición a las demás corrientes, el materialismo cultural acomete la tarea de definirse en un plano teórico general. En cuanto conjunto de principios y elementos programáticos de carácter teórico y metodológico, Harris asocia su proyecto a la noción kuhniana de paradigma. En su obra El Materialismo cultural, se propone una descripción detallada y sistemática de los objetivos reales y presupuestos básicos que conforman, junto a los resultados sustantivos, la estructura de un programa científico de investigación, o paradigma.

Esta explicitación es necesaria a modo de prerrequisito para una elección racional entre opciones paradigmáticas referidas al campo de la antropología en calidad de competidores rivales. Inspirado también en ideas de Popper, Harris cree que una elección racional entre paradigmas es posible, con tal que se remplacen los programas rudimentarios e inconscientes por descripciones completas y exhaustivas de los mismos. Tales descripciones permitirán evaluar a priori la simplicidad e inteligibilidad de los diversos paradigmas, pudiéndose optar por aquel que mejor "administre" la promesa de realización del ideal de la ciencia.

La meta del materialismo cultural, esto es, el horizonte de realización y concreción de un ideal científico en antropología, consiste en explicar el origen, mantenimiento y cambio del inventario global de diferencias y semejanzas socio culturales. Ahora, con la vinculación del objeto de la antropología a un programa reducido y delimitado por los términos de la ciencia empírica se pone en entredicho la relevancia de los 
debates metateóricos. Harris enfatiza el hecho de que ninguna crítica es suficiente por sí misma para desmontar un programa de investigación. Esta negativa tiene por objeto la descalificación de ciertas discusiones más o menos fronterizas y filosóficas desde las cuales se intenta redefinir el objeto y los fines de la ciencia del hombre, poniendo en juego categorías que no están sustentadas en la necesaria evaluación del material etnográfico y, lo que es aún peor, desde las cuales se borra como de un brochazo todo el trabajo de consolidación conceptual e investigación empírica que ha acreditado la antropología en el concierto exigente de las disciplinas positivas.

Nuestro propósito no es pormenorizar aquí sobre este asunto, acerca del cual diremos sólo que atañe al problema global de la diferenciación entre ciencia y filosofía y al más específico de los límites del conocimiento antropológico que implica, por tanto, una reflexión más amplia y apuntalada en una conceptualización epistemológica en torno a la cual subsiste el debate abierto. En la propia doctrina de Harris, por lo demás, hay bosquejado ya un plan en ese sentido. Harris, en efecto, intenta hacer explícitos sus propios fines, métodos y principios, sobre la base de un análisis crítico de los por él llamados paradigmas alternos en antropología. Este análisis constituiría una región común a la epistemología y la antropología, donde el debate lógico y metodológico entroncaría con la problematización filosófica en torno al objeto de una disciplina que responda a las inquietudes más elementales y urgentes respecto a la naturaleza humana. Con ello se lograrían establecer unas normas básicas para regular las discusiones y unificar los criterios entre filósofos y científicos, cuyo intercambio en este terreno acusa una grave afinidad con el diálogo de sordos.

Las siguientes consideraciones, lejos de pretender remover la problemática total del programa antropológico de Harris, centrarán su atención en el rasgo determinista que el autor defiende de modo explícito como un rasgo esencial ${ }^{4}$. El significado interno y las implicaciones concretas del uso y aplicación de dicho principio han sido ya expuestos en sus rasgos principales. A continuación abordaremos su significado más general de cara a lo que representa el determinismo como proyecto explicativo de la conducta humana y la subsecuente dificultad referida a la libre direccionalidad de la voluntad individual. Esto no debe entenderse, en modo alguno, como proyecto de erección de una cierta aduana metacrítica para una disciplina cuya positividad admitimos con parecidas razones que su mentor principal. Se trataría, más bien, de aprovechar la reviviscencia de un tema tan atractivo como el determinismo para recrear viejos y tenaces motivos de reflexión filosófica.

El materialismo cultural resulta, pues, de la unificación de los principios materialistas con los principios evolucionistas de corte darwiniano. Sin embargo, se trata de un materialismo especial por cuanto sustituye la teleología del materialismo histórico por la tradición explicativa de corte causal, propia de la ciencia natural y empírica. Igualmente, niega la dialéctica como tesis objetivada aplicable al desarrollo de la sociedad, lo mismo que como método de interpretación histórica.

En tal sentido, el determinismo tecnoecológico y tecno-económico, en tanto se comprenda como un análogo del principio darwinista de la selección natural, permite ser usado y entendido no en el sentido problemático de conjunto de leyes específicas de la evolución cultural sino como estrategia cuya aplicación permite comprender causal y nomotéticamente

4 “La Adopción sistemática del criterio del determinismo materialista constituye la condición de posibilidad de una explicación científica de los fenómenos socioculturales". (Harris, 1981, p.4). 
los fenómenos sociohistóricos. La adopción del determinismo evolucionista representa, en últimas, "conferir prioridad al estudio de las condiciones materiales de vida igual que el principio de la selección natural da prioridad al estudio de las diferencias de eficacia reproductora" (Harris, 1981, p.3).

Como propuesta reductiva de corte causal de las conductas el materialismo cultural representa una estrategia de distensión sistemática de la subjetividad, y ello explica ciertos rendimientos del paradigma en la perspectiva crítica frente a las ideologías oscurantistas, mistagógicas e irracionales. En ello, sea dicho de paso, se revela cierta fidelidad de Harris con el marxismo; de otro lado, aquella suspensión reductiva, en la medida en que se la interprete mecánicamente, generará resistencias automáticas, contra las que Harris siempre vuelve en actitud beligerante.

En estrecha relación con su vocación empírica, encontramos en el materialismo cultural una segunda instancia crítica dirigida, esta vez, contra los modelos explicativos basados en procedimientos que acotan, dentro del campo de los fenómenos, un determinado grupo de acontecimientos privilegiados en calidad de entes modélicos, términos y relaciones, entre otros. El grueso de la crítica de Harris se dirige, en este sentido, contra el programa de la antropología estructuralista de Levi-Strauss y sus discípulos. En dicha perspectiva se tiende a asimilar el objeto a las propias condiciones subjetivas. Así, en cuanto lo que se pretenda hallar sean las leyes de la conciencia simbólica, es decir, la propia estructura subjetiva, el acuerdo entre la teoría y el fenómeno se daría por descontado. Esto indicaría que, al considerar la cultura como sistema de comunicación, los fenómenos se determinan como mensajes $y$, en cuanto tales, las formas reales de vida tienden a asimilarse al código subyacente prestablecido. Entre el fenómeno y el substrato objetivo no media más que un proceso de decodificación; pero, al acentuarse y hacerse explícito además el carácter dialéctico de esta correspondencia (Levi-Strauss, 1989, p.119), no se lograría otra cosa que justificar a posteriori el análisis diseñado a priori, ya que cualquier desfase entre estas instancias (fenómeno y substrato objetivo) es siempre subsanable por el recurso al contexto. En este caso, se trata de un recurso tal que deja la sensación de crasa manipulación del dato etnográfico, donde se le hace valer como instancia de legitimación opcional de carácter negativo antes que como instancia positiva de autocorrección y control de la teoría, según lo exigen los cánones científicos.

Esto por lo que respecta a la crítica de la antropología estructural desde la óptica del materialismo naturalista de Harris. Por otra parte, dado el carácter determinista y el ideal nomotético pregonados, el materialismo cultural se enfrenta a dos dificultades en cierto modo metafísicas. La primera tiene que ver con el problema de la libertad y la segunda con el de la historia.

Veamos primero el asunto de la libertad. La sujeción a los cánones de la ciencia empírica supone una idea totalmente naturalizada de las conductas y proyecta además la imagen de una conciencia cuyos actos individuales están objetivamente determinados. Para ser precisos, sin embargo, digamos que tal concepción no es ni originaria ni privativa de la ciencia y que tiene antecedentes bastante claros en el racionalismo y en el panteísmo, no sólo en el moderno (Bruno, Spinoza, Shelling, Hegel) sino en el antiguo, asociado a la especulación cosmofísica (Demócrito, Epicuro, Lucrecio). 
El proyecto de sometimiento de los actos humanos al régimen de explicación causal no implica la negación de la libertad y, en verdad, nadie sostendría eso. Lo que se plantea es más bien la dificultad inherente a las expectativas sistemáticas de explicación a la vista de la innegable condición de la conciencia individual en cuya base admitimos la elección incondicionada de un vastísimo conjunto de "movimientos" a partir de los cuales se ha configurado la imagen de su autonomía.

El materialismo cultural sería, pues, sospechoso de proyectar una finalidad aporética en sí misma: pretender hacer analíticamente explícito, es decir, determinado, lo que constituye el órgano mismo de la indeterminación: la libre elección de la voluntad. Sin embargo, el paradigma de Harris puede eludir satisfactoriamente esta dificultad en la medida en que el programa de explicitación no se perfila sobre el horizonte ontológico del primado absoluto del ser o de la conciencia. Es esto lo que significa la aseveración de Harris en el sentido de que al materialismo cultural le basta con postular el determinismo como estrategia de obtención de conocimiento válido en la dirección de la validez científica, es decir, de la contrastabilidad y la intersubjetividad, tanto a partir de las conductas como de los sistemas ideativos.

No se trataría, por cierto, de establecer un programa de sustentación formal y positiva, de base naturalista, del primado del ser sobre la conciencia, lo cual es absurdo, sino más bien de rastrear metódicamente, en el terreno de sus objetivaciones, los fundamentos reales de la determinabilidad de la conciencia, aunque se reconozca utópica y mítica la vía analítica de su explicitación total.

Para llamarlo de algún modo, éste sería un materialismo restringido, que puede salvarse de la dificultad a la que se ha aludido por cuanto constituye una recuperación formal y no sustantiva del cientifismo. A esto precisamente es a lo que equivale su negativa de hacer valer el privilegio de las cadenas motivacionales de la conducta como tesis ontológica y la decisión de concebirlas, en cambio, como un postulado estratégico. Esta decisión filosófica define también para el materialismo cultural su posición metodológica, positiva y empírica pero no formalista. Esto indica que el determinismo tecno-económico y tecnoecológico no puede pretender valer como un axioma indefinible y asimilable, por ende, a los principios de la derivación matemática, al final de cuyas cadenas analíticas deberían hallarse los actos discretos de la conciencia subjetiva. Esta propensión formalizante pertenece más bien al estructuralismo de Levi-Strauss, en el cual halla Piaget el paradigma (al menos como proyecto) de disciplina deductiva en las ciencias humanas (Piaget, 1974, p.123).

En cuanto el materialismo cultural ofrezca un tratamiento crítico y no mecánico de los procesos, de eficacia muchas veces retroactiva, que son tipificables como fenómenos de base subjetiva, esto es, prácticas puramente mentales, con una dinámica propia y que alcanzan un rango creciente de objetividad conforme crece la complejidad de las relaciones sociales y políticas, puede decirse que no es incompatible con la idea, en todo sentido digna de defenderse, de que son también y decisivamente las ideas las que constituyen los motores de la transformación histórica, y no una mera cadena sinfín de motivaciones expresables en los puros términos de las leyes de circulación energética.

Es innegable, por otro lado, que el conocimiento de la base motivada de la conducta es un requisito insoslayable de cualquier voluntad de racionalización 
social. Además, el materialismo cultural no suscribe la total irreversibilidad de las leyes que gobiernan los conglomerados humanos, pues no se trata de leyes mecánicas, ni afirma su condición ineluctable conforme al antecedente arcaico de un orden preestablecido, regimentado por una voluntad omnisciente e inexorable. Antes bien, la posibilidad de que los hombres puedan sustraerse a las consecuencias funestas que derivan del manejo ciego, exclusivista, irracional y pendenciero de la sociedad, tiene que arraigar en un claro conocimiento de sus leyes y de la naturaleza de sus constituyentes decisivos: los seres humanos.

Repetidas veces encontramos en las obras de Harris alusiones en contra de toda actitud que se obstine en defender la dignidad de la condición humana sobre el supuesto de una libertad meramente aparente, desde la cual se intenta desconocer la base legalmente motivada de la conducta y de la acción social. Dichas concepciones parecen concebir la existencia social como equivalente a un reino de la pura fatalidad, con lo cual alimentan las tendencias al sometimiento ciego ante la marcha forzosa de una sociedad que, precisamente por decisiones nada fatales, se conduce para que permanezcan inamovibles las reparticiones injustas de privilegios, cargas e imposiciones.

El materialismo cultural no cifra tampoco su dignidad teórica en una libertad humana ilusoria, que a menudo no es otra cosa sino la fantasmagoría resultante de anteponer la subjetividad como medida de lo humano. Al contrario, reconoce las determinaciones objetivas de la acción, incluso de las acciones deliberadas, y no ve en ello abdicación alguna respecto del principio de autonomía individual como tampoco respecto de la dignidad metafísica que confiere al hombre su capacidad de orientarse conforme a fines e incluso de forjar esos mismos fines.

Es preciso indicar también que la no suscripción del método dialéctico y la asunción concomitante de la forma del cientifismo permiten establecer con mayor claridad la noción marxiana de determinación en última instancia, diferenciando la determinabilidad social de la conciencia, como propuesta de racionalización del movimiento histórico, de la tesis problemática del primado ontológico del principio material, con su consecuencia ineludible de la subordinación del rendimiento subjetivo, por donde se cuela inevitablemente un dogmatismo indeseable.

El materialismo cultural, según expresa confesión de Harris, se postula también como alternativa de una ciencia de la historia. En este particular es concebible otra dificultad de índole metafísica y que tiene que ver con el compromiso determinista y nomotético de las explicaciones derivadas de dicho paradigma, de cara a lo que se tiene como evidente en la perspectiva de la cognoscibilidad de los sucesos humanos e históricos, a saber: su impredecibilidad. Este problema es similar al planteado por Popper en su obra La miseria del historicismo (1981) en calidad de objeción insalvable contra la cognoscibilidad científica del movimiento histórico.

¿Qué tendría que decir el Materialismo cultural frente a la acusación de fracaso lógico de su ideal predictivo, siendo que se ha propuesto llevar a la concreción disciplinar las expectativas de una ciencia de la historia? En primer lugar, la negativa formal de todo determinismo, a partir del señalamiento de la imposibilidad lógica de predicciones en torno a los actos humanos, constituye una objeción a su principio arquitectónico, la cual puede ser fácilmente refutable. EI determinismo del materialismo cultural 
es expresamente probabilista, tal como se consigna en la formulación misma del primado de la infraestructura. Esto significa que la posibilidad de predicción puntual del futuro no es aquí fundamento constructivo. Nótese además que, tras la exigencia de una satisfacción digamos "atómica" de la contingencia en los órdenes de la conducta y de la acción humana, yace un empirismo epistemológico a partir del cual no puede seguirse nada. Cuando se antepone la objeción de la impredecibilidad a la posibilidad de la determinación causal de los eventos se procede de acuerdo con una identificación básica inaceptable: la que proyecta la deductibilidad en su más pura expresión formal, esto es, allí donde el tiempo es ya una recusación suficiente de la identidad, sobre los acontecimientos contingentes, lo cual implica exigir el acuerdo observacional e intuitivo como única modalidad constructiva de explicación causal, cosa que ninguna ciencia particular hace ni puede hacer.

De muy diversa índole son los análisis de factibilidad, los análisis semiológicos de la diagnosis médica, de la prognosis climática $y$, en general, todas las estrategias que se estatuyen según el esquema básico de la "adivinación" del futuro, las cuales se fundan en modelos estadísticos y probabilísticos que constituyen precisamente la solución y revocatoria efectiva de la objeción fundada en la impredecibilidad. Finalmente, es claro que, conforme a las exigencias sistemáticas de semejante "principio", la mayoría de las ciencias sociales irían a parar a la hoguera que Hume recomendara para toda "vana metafísica" y a la que condenó sin advertirlo buena parte del saber positivo que ya era inmune a la acción de las llamas.

En segundo lugar, esta negativa formal del determinismo merece ser analizada también en lo que representa más allá de su dimensión gnoseológica. El argumento de Popper es una elaboración técnica y bastante radical de una constelación ideológica que, lejos de ser una postura sólo epistemológica, alimenta, frente a los asuntos humanos, un conjunto de ideas que han sido aprovechadas en el negativo sentido de hacer que signifiquen que el curso de los sucesos que conforman el movimiento de la sociedad y de la historia sea sencillamente la expresión del puro azar o de fuerzas impenetrables e ineluctables. Como supuesto ideológico subyace a todas las formas de irracionalismo a través de las cuales prosperan las fuerzas por las que se perpetúa, se disculpa, se tolera o ingenuamente se colabora con el principio de dominio y de barbarie, y al contrario, los ideales de equidad humana han sido siempre solidarios de expectativas ilustradas, esto es, de la afirmación de la cognoscibilidad de los sucesos como fundamento de una acción racionalizadora que permita la liberación y la desalienación efectiva de los hombres.

Ahora bien, frente a la sospecha de que la reivindicación de la racionalidad científica esconde ella misma el viejo motivo de la dominación $y$, en el campo de la antropología, el mismo vulgar etnocentrismo contra el que ha comenzado a luchar la disciplina como requisito de su propia emancipación teórica, hay que decir lo siguiente: el materialismo cultural suscribe el determinismo material no sobre el principio del mero rendimiento, fundado en los criterios de la manipulación técnica o en las falacias del desarrollismo, sino a la luz del postulado de optimización y regulación racional de los intercambios de la especie con el medio y consigo misma. De acuerdo con ello, lo que se afirma no es un afán de clausurar la historia colocando el colofón de algún credo infalible como guía y menos el impersonal e inhumano postulado de 
racionalización del que esté ausente un sentido ético al que se subordine todo movimiento de regulación.

Así, pues, la afirmación de la legalidad determinada de la evolución social y cultural y la suscripción del primado del componente material de tal determinación no se opone a la idea metafísica de libre perfectibilidad de la condición humana; antes bien, lo afirma, pero no desde el mero voluntarismo reflexivo y como un "factum", sino desde las exigencias de la objetividad, como un compromiso histórico, mediado precisamente por la premisa que representa el reconocimiento de la objetiva legalidad del curso de la sociedad y de la historia. Adviértase además que este curso no equivale de suyo a "ascenso" dignificador y no puede interpretarse como sinónimo de progreso.

El materialismo cultural se definiría entonces como proyecto de asunción explícita de la razón como guía de la existencia histórica, antes que como un racionalismo a ultranza. Desde esta perspectiva el evidente mal de la sociedad moderna aparece no como el fruto de un supuesto exceso de razón sino como todo lo contrario, como la consecuencia de la ausencia de razón en el orden de la existencia colectiva (Harris, 1986, pp.222227).

De otro lado, la idea que una orientación racional es posible y humanamente deseable no tendría por qué equivaler a una forma etnocentrista de teorización antropológica. La idea de una interpretación naturalista de los fenómenos socio culturales no puede hacerse equivalente a la violencia etnocentrista, herencia a su vez de la violencia colonialista.
La debida sustentación de este punto de vista exige una argumentación que desborda los objetivos del presente artículo, con lo cual se deja abierta la posibilidad investigativa, que afronte los vacíos y nuevos tópicos reflexivos en el proyecto en curso de antropología y epistemología. Sin embargo, para finalizar se señala el hecho, a este respecto pertinente, que el materialismo cultural pretende aportar una explicación naturalista, es decir, universalmente aceptable, con respecto a la efectiva y de verdad condenable violencia a la que son sometidas las culturas dominadas. En esa perspectiva, la cuestión de la identidad cultural, entendida como inalienabilidad y pristinidad a-conceptual del alma de los pueblos, frente a cuya pureza la mirada exterior sería desvirtuante y violenta, aunque no se sigue de este enfoque, sería un juego de niños, comparado con el avance "civilizador" de la cultura capitalista y de la planetarización de la sociedad técnica y de masas, sobre los derroteros objetivamente indeseables de la geopolítica moderna.

\section{Referencias}

Harris, M. (1981). El desarrollo de la teoría antropológica. Madrid: Ed. Siglo XXI.

Harris, M. (1982). El materialismo cultural. Madrid: Alianza.

Harris, M. (1986). Vacas, cerdos, guerras y brujas. Los enigmas de la cultura. Madrid: Alianza.

Levi-Strauss, C. (1986). Antropología estructural II. Madrid: Ed. Siglo XXI.

Piaget, J. (1974). El estructuralismo. Barcelona: OikosTAU S.A.

Popper, K. (1981). La miseria del historicismo. Madrid: Alianza Editorial. 